\title{
COMPARISON BETWEEN DIFFERENT PARAMETERS IN EARLY DETECTION OF LV SYSTOLIC DYSFUNCTION IN PATIENTS WITH CHRONIC STABLE ANGINA
}

\author{
By \\ Taghreed Abd El-Rahman Ahmed and Mona Naiem Ali Hassan \\ Cardiology Department, Faculty of medicine (Girls), Al-Azhar University, Cairo, Egypt \\ E-mail: tahersalman2017@ gmail.com
}

\begin{abstract}
Background: Ischemia plays a pivotal role in the development and progression of both types of HF (HFrEF \& HFpEF). Strain echocardiography, performed by using the speckle tracking technique, can identify subclinical left ventricular dysfunction before left ventricular ejection fraction declines.
\end{abstract}

Objective: To assess left ventricular systolic function by different noninvasive imaging modalities in patients with chronic ischemic heart disease.

Patients and methods: This study was done at Al Zahra University Hospital from November 2017 till January 2020. It included 80 patients (group I) with chronic ischemic heart disease (IHD), they were 60

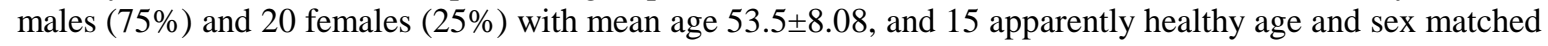
subjects as a control group (group II), they were 11(73.3\%) females and 4(26.7\%) males with mean age 53.2 \pm 7.7 . Group I was further sub-classified to impaired and preserved according to their LVEF assessed by 2D echo, speckle tracking echo global longitudinal strain (STE-GLS), tissue Doppler imaging (TDI) strain, TDI Sa and gated SPECT LVEF.

Results: The ischemic (group I) showed a statistically significant decrease in the average Sa $5.45 \pm 1.69$ VS group II $8.7 \pm 0.73$ (P value $<0.001$ ), lower LVEF 2D echo in group I $46.3 \pm 9.68$ compared to group II $58.9 \pm 4.68$ ( $\mathrm{P}$ value $<0.001$ ), decrease in gated SPECT LVEF in group I 56.4 \pm 13.98 VS group II $67.067 \pm 5.66$ ( $\mathrm{P}$ value $<0.001$ ), statistically significant reduction in left ventricular global longitudinal strain (LV-GLS) $(-13.89 \pm 18.99)$ in group I vs $-21.24 \pm 1.23$ in group II (P value $<0.05)$. The highest percentage of patients with impaired LV systolic function were those who assessed by STE-GLS (74 patients 92\%), 72 patients (90\%) impaired LVEF by TDI Sa, 60 patients (75\%) impaired LVEF by 2D echo, and by TDI strain, and 22 patients (27.5\%) impaired LVEF by gated SPECT.

Conclusion: STE is considered the most sensitive method in assessment of subclinical LV systolic dysfunction and is considered superior to other methods.

Keywords: CAD; 2D STE; TDI; gated SPECT.

\section{INTRODUCTION}

Left ventricular functional parameters are useful diagnostic and prognostic indicators for the appropriate management of patients with ischemic heart disease (Becker et al., 2012). Quantification of regional and global motion and thickening of the left ventricle might be helpful in the assessment and follow-up of patients with myocardial dysfunction and in evaluating the efficacy of therapeutic interventions, whether medical or invasive therapy in these patients (Mujtaba et al., 2013). 
Echocardiographic,

magnetic resonance imaging (MRI), and scintigraphic studies have revealed that regional and global myocardial motion and wall thickening can be quantitatively assessed using these techniques (Migrino et al., 2015).

The aim of this study was to assess left ventricular systolic function by different noninvasive imaging modalities in patients with chronic ischemic heart disease.

\section{PATIENTS AND METHODS}

Study cohort: This study was conducted on 80 patients (group I) with chronic ischemic heart disease. They were collected from those who were referred for myocardial perfusion imaging at $\mathrm{Al}$ Zahraa University Hospital. Another group of 15 healthy age matched individuals that were collected retrospectively from cases candidate for MPI study and their studies were negative for myocardial ischemia, had been enrolled as a control group (group II). The study was conducted during the period from November 2017 till January 2020.

We excluded patients with acute coronary syndrome, significant valve disease, patients with arrhythmias (AF, frequent PVCs or APCs), and patients with poor echo window. Written consents have been signed according the institutional ethical committee recommendations.

Method: Detailed history, medical therapy, 12 lead ECG, and stress exercise ECG were done in all cases. Also, weight, height and body mass index (BMI) were recorded.
Trans-thoracic echocardiography (TTE) was performed using Vivid-E9 GE system with tissue Doppler imaging (TDI) capability. We used multi frequency (2.53.5 $\mathrm{MHz}$ ) matrix probe M3S with simultaneous ECG physio signal displayed with all recorded echo images and loops. For image acquisition, 3cardiac cycles were taken in each view with the patient holding his or her breath. All images were digitally stored for off line analysis (EchoPAC.GE VERSION 113-202). All parameters were taken according to the ASE standards and recommendations of the European Association of Cardiovascular Imaging.

Assessment of the $\mathbf{L V}$ was done using 2D guided M-mode echocardiography to assess Left ventricle end diastolic dimension (LVEDD), Left ventricle end systolic dimension (LVESD), Inter ventricular septal dimension (IVSD), Left ventricular posterior wall dimension (LVPWD) and Fractional shorting (FS). 2D echocardiography was used to assess Ejection fraction (EF\%) (Simpson method), Segmental wall motion abnormalities and regional

Wall Motion Score Index (WMSI) was calculated. Pulsed wave Doppler (PWD) was performed to assess transmitral maximal Velocities Peak (E), peak (A) velocities, the $\mathrm{E} / \mathrm{A}$ ratio and deceleration time of early mitral flow and using TDI to evaluate peak ( $\mathrm{S}^{\prime}, \mathrm{E}^{\prime}$ and $\mathrm{A}^{\prime}$ velocities) and global LV strain.Two-dimensional speckle tracking analysis performed on LV was obtained in apical 4, 2and 3 chambers, global strain assessed by averaging strain of all segments.Myocardial perfusion imaging using 99mTc-sestamibi were done to 
assess LV systolic function, systolic and diastolic volumes, to detect ischemia and to measure peak empting rate.

Statistical analysis: Numerical variable was expressed as mean and standard deviation (SD); the following statistical tests were used for analysis of data by SPSS version 19: Independent $t$ test: for testing statistical significant difference between means of the two groups in each classification Pearson's correlation test with the determination of the correlation coefficient ( $r$ ) to test a positive or negative relationship between two variables. $\mathrm{P}$ Value less than 0.05 was considered statistically significant. Value less than 0.2 was considered very weak correlated. 0.2-0.4 (weak correlation), 0.4-0.6 (moderate correlation), $0.6-0.8$ (strong correlation), and $>0.8$ (very strong correlation).

\section{RESULTS}

This study included 80 patients (group I) with chronic ischemic heart disease, they were (60 males and 20 females) from those who were referred for myocardial perfusion imaging unit at Al-Zahraa University Hospital in addition to 15 healthy subjects (group II) as a control group (4males and 11 females) with mean age $53.5 \pm 8.08$ for the ischemic group and $53.2 \pm 7.7$ for the control group ( $\mathrm{P}$ value $=0.902$ ).

The demographic data of the patients group (group I) were: 52 diabetics, 40 patients hypertensive, 58 dyslipidemic, 20 smokers, 26 ex-smokers and 18 had positive family history. As regard symptoms, 66 patients complained of chest pain, and 18 patients complained of dyspnea. As regard ECG, 50 patients had abnormal resting ECG.

There were statistically highly significant differences between group I and group II as regard: sex, DM, HTN, DLP, smoking, FH, Chest pain, SBP, DBP and ECG abnormality ( $\mathrm{P}$ value < 0.05). There was a statistically significant increase in LVEDD, SWMA 2D echo score, and AO diameter and a lower LVEF 2D echo in group I compared to group II ( $\mathrm{P}$ value $<0.001)$, and significant increase in LVESD, and LA diameter in group I compared to group II ( $\mathrm{P}$ value < 0.05). There were non-significant differences between the two groups as regard IVSD, FS, LA/AO, MV E-vel, MV A-vel (Table 1). 
Table (1): Comparison between the two groups as regard the conventional echocardiographic parameters

\begin{tabular}{|c|c|c|c|}
\hline Groups & Patient $\mathrm{N}=80$ & Control N= 15 & $P$ value \\
\hline Parameters & & & F value \\
\hline LVEDD (mm) & $5.37 \pm 0.65$ & $4.5 \pm .61$ & $<0.001$ \\
\hline LVESD (mm) & $3.47 \pm 0.94$ & $2.86 \pm .26$ & 0.015 \\
\hline IVSD (mm) & $9.8 \pm 0.27$ & $9.1 \pm .07$ & 0.351 \\
\hline FS $(\%)$ & $35.57 \pm 11.36$ & $39.6 \pm 3.9$ & 0.187 \\
\hline LVEF-2D echo (\%) & $46.3 \pm 9.68$ & $58.9 \pm 4.68$ & $<0.001$ \\
\hline $\mathrm{AO}(\mathrm{cm})$ & $3.1 \pm 0.46$ & $2.68 \pm 0.46$ & 0.002 \\
\hline LA (cm) & $3.6 \pm 0.67$ & $3.2 \pm 0.18$ & 0.034 \\
\hline LA/AO & $1.1 \pm 0.28$ & $1.1 \pm 0.15$ & 1 \\
\hline MV E-vel .(mm/s) & $6 \pm 0.18$ & $6 \pm 0.11$ & 0.981 \\
\hline MV A-vel $(\mathrm{mm} / \mathrm{s})$ & $0.78 \pm 0.85$ & $0.58 \pm 0.13$ & 0.167 \\
\hline SWMA score & $1.4 \pm 0.46$ & $1 \pm 0$ & 0.001 \\
\hline
\end{tabular}

There was a statistically significant decrease in the average $\mathrm{Sa}$ in group I compared to group II $(\mathrm{P}<0.001)$, significant lower average strain value measured by TDI in group I compared to group II ( $\mathrm{P}$ value <0.05), significant decrease in STE GLS in group I compared to group II ( $\mathrm{P}$ value <0.05), significant increase in LVEDV $(\mathrm{P}$ value $=0.029) \mathrm{a}$ significant increase in SWMA score index ( $\mathrm{P}$ value $<0.001$ ) in group I compared to group II, significant decrease in LVEF, and PER in group I compared to group II $(\mathrm{P}$ value $<0.001)$ (Table 2$)$.

Table (2): Comparison between the two groups as regard TDI Sa, Avg TDI strain, STE-GLS and LV function assessed by gated SPECT.

\begin{tabular}{|c|c|c|c|}
\hline Groups & & & \\
\hline Parameters & Patients $\mathrm{N}=80$ & Control $\mathrm{N}=1 \mathrm{5}$ & P value \\
\hline $\operatorname{Avg~Sa}(\mathrm{mm} / \mathrm{s})$ & $5.45 \pm 1.69$ & $8.7 \pm 0.73$ & 0.0001 \\
\hline $\operatorname{Avg} \operatorname{Ea}(\mathrm{mm} / \mathrm{s})$ & $6.1 \pm 2.2$ & $6.56 \pm 1.3$ & 0.455 \\
\hline $\operatorname{Avg~Aa}(\mathrm{mm} / \mathrm{s})$ & $6.47 \pm 1.84$ & $7.74 \pm 2.47$ & 0.087 \\
\hline Avg TDI strain\% & $-16.6 \pm 4.56$ & $-19.3 \pm 3.76$ & 0.034 \\
\hline STE- GLS (\%) & $-13.89 \pm 18.99$ & $-21.24 \pm 1.23$ & 0.02 \\
\hline LVEDV (ml) & $118 \pm 42.15$ & $92.7 \pm 17.6$ & 0.029 \\
\hline LVESV (ml) & $56 \pm 39$ & $37.27 \pm 9.13$ & 0.073 \\
\hline LVEF \% & $56.4 \pm 13.98$ & $67.067 \pm 5.66$ & 0.006 \\
\hline SWMA score & $1.34 \pm 0.316$ & $1 \pm 0$ & 0.005 \\
\hline PER & $2.89 \pm 0.83$ & $3.6 \pm 0.5$ & 0.0001 \\
\hline
\end{tabular}

We assessed the left ventricular systolic function for all patients (No 80) by different echo modalities (conventional echo, TDI velocity, TDI strain, STE GLS) and gated SPECT scan. We divided the ischemic group (group I) into two subgroups: (1) impaired and (2) preserved according to their LVEF by 2D echo, STE-GLS, average TDI strain, Avg Sa and gated SPECT LVEF (A,B,C,D\& E respectively). Eighteen patients were found to have impaired LV systolic function by all methods (LVEF by 2D echo, STE-GLS, average TDI strain, Avg $\mathrm{Sa}$ and gated SPECT), 28 patients were found to be impaired LV systolic function by four methods, 16 patients were found to impaired LV systolic function by three 
methods, 18 patients were found to impaired LV systolic function by two methods.

Demographic data of group A1 showed that there were 44 patients diabetics, 36 patients had hypertension, 48 patients had dyslipidemia, 20 male patients were smokers, 18 patients Exsmoker and 16 patients had positive $\mathrm{FH}$. Group A2 showed that there were 14 patients were diabetics, 4 patients had hypertension, 10 patients had dyslipidemia, 8 patients Ex-smoker and 2 patients had positive FH. There was only significant increase in hypertensive patients among patients with impaired 2D echo LVEF (Group 1A) (P value $=0.028$ ), (Table 3).

Group 1B showed that there were 54 diabetics patients, 40 patients had hypertension, 54 patients had dyslipidemia, 20 male patients were smokers, 24 patients Ex-smoker, 18 patients had positive $\mathrm{FH}, 60$ patients complained of chest pain, 16 patients complained of dyspnea and 44 patients had abnormal resting ECG, Group 2B showed that There were 4 patients diabetics, 24 patients had dyslipidemia , 4 patients ex-smoker , 6 patients complained of chest pain, 2 patients complained of dyspnea and 6 patients had abnormal resting ECG . There were no statistically significant differences between the two groups as demographic data, (Table 3).

In group $1 \mathrm{C}$ there were 38 patients diabetics, 30 patients had hypertension, 40 patients had dyslipidemia, 16 male patients smokers, 18 patients ex-smoker, 10 patients had positive $\mathrm{FH}, 46$ patients complained of chest pain, 16 patients complained of dyspnea and 18 patients had abnormal resting ECG, Group 2C showed that there were 20 patients diabetics, 10 patients had hypertension, 18 patients were dyslipidemia and 4 male patients were smokers, 8 patients Ex- smoker, 8 patients had positive $\mathrm{FH}, 20$ patients complained of chest pain, 2 patients complained of dyspnea and 8 patients had abnormal resting ECG . There were no significant differences between the two groups as regard their demographic data except for statistically significant increase in diabetic patients among patients with impaired function as assessed by TDI strain (Group 1C) (P value $=0025)$, (Table 3$)$.

Group 1D showed that there were 50 patients diabetics, 34 patients had hypertension , 50 patients had dyslipidemia, 18 male patients were smokers, 22 patients Ex-smoker, 18 patients had positive $\mathrm{FH}, 62$ patients complained of chest pain, 14 patients complained of dyspnea and 44 patients had abnormal resting ECG, Group 2D showed that there were 8 patients diabetics, 6 patients had hypertension , 8 patients had dyslipidemia\& 2 male patients smokers, 4 patients ex-smoker and 4 patients complained of chest pain, 2 patient complained of dyspnea and 6 patients had abnormal resting ECG . There were no significant differences between the two groups as regard their demographic data, (Table 3).

In group $1 \mathbf{E}$ there were 18 patients diabetics, 16 patients had hypertension, 20 patients had dyslipidemia, 12 male patients were smokers, 8 patients exsmoker, 4 patients had positive $\mathrm{FH}, 14$ patients complained of chest pain, 10 patients complained of dyspnea \& 16 patients had abnormal resting ECG, In Group 2E there were 40 patients diabetics, 24 patients had hypertension, 38 patients had dyslipidemia and 8 male patients were smokers, 18 patients exsmoker, 14 patients had positive $\mathrm{FH}, 52$ patients complained of chest pain, 8 patients complained of dyspnea and 32 patients had abnormal resting ECG . There were statistically significant 
increase in number of smokers $)(\mathrm{P}$ value $=$ 0.009), and statistically significant increase in number of patients complaining of chest pain) $(\mathrm{P}$ value $=$ 0.053 ) among patients with impaired gated SPECT LVEF (Group 1E), and increase in number of patients complaining of dyspnea in patients with preserved gated SPECT LVEF (group 2E) $(\mathrm{P}$ value $=0.032)($ Table 3$)$.

Table (3): Comparisons between demographic data in all sub groups

\begin{tabular}{|c|c|c|c|c|c|c|c|c|c|c|c|}
\hline \multirow{2}{*}{\multicolumn{2}{|c|}{$\begin{array}{ll}\text { Variables } & \text { Groups } \\
\end{array}$}} & \multirow{2}{*}{\multicolumn{2}{|c|}{ Group A }} & \multirow{2}{*}{\multicolumn{2}{|c|}{ Group B }} & \multicolumn{2}{|c|}{ Group C } & \multicolumn{2}{|c|}{ Group D } & \multirow{2}{*}{\multicolumn{2}{|c|}{ Group E }} \\
\hline & & & & & & & & & & & \\
\hline & & A1 & A2 & B1 & B2 & C1 & $\mathrm{C2}$ & D1 & D2 & E1 & E2 \\
\hline \multirow{2}{*}{ Sex } & Female & 12 & 8 & 18 & 2 & 16 & 4 & 20 & $\mathbf{0}$ & 2 & 18 \\
\hline & Male & 48 & 12 & 52 & 4 & 44 & 16 & 52 & 8 & 20 & 40 \\
\hline \multirow{2}{*}{ DM } & No & 16 & 6 & 20 & 2 & 22 & $\mathbf{0}$ & 22 & $\mathbf{0}$ & 4 & 18 \\
\hline & Yes & 44 & 14 & 54 & 4 & 38 & 20 & 50 & 8 & 18 & 40 \\
\hline \multirow{2}{*}{ HTN } & No & 24 & 16 & 34 & 6 & 30 & 10 & 38 & 2 & 6 & 34 \\
\hline & Yes & 32 & 4 & 40 & $\mathbf{0}$ & 30 & 10 & 34 & 6 & 16 & 24 \\
\hline \multirow{2}{*}{$\begin{array}{c}\text { Dyslipidemi } \\
\text { a }\end{array}$} & No & 12 & 10 & 20 & 2 & 20 & 2 & 22 & $\mathbf{0}$ & 2 & 20 \\
\hline & Yes & 48 & 10 & 54 & 4 & 40 & 18 & 50 & 8 & 20 & 38 \\
\hline \multirow{3}{*}{ Smoking } & No & 22 & 12 & 30 & 4 & 26 & 8 & 32 & 2 & 2 & 32 \\
\hline & Yes & 20 & $\mathbf{0}$ & 20 & 0 & 16 & 4 & 18 & 2 & 12 & 8 \\
\hline & X-smoker & 18 & 8 & 24 & 2 & 18 & 8 & 22 & 4 & 8 & 18 \\
\hline \multirow{2}{*}{ FH } & Negative & 44 & 18 & 56 & 6 & 50 & 12 & 54 & 8 & 18 & 44 \\
\hline & Positive & 16 & 2 & 18 & 0 & 10 & 8 & 18 & 0 & 4 & 14 \\
\hline
\end{tabular}

Comparisons between each parameter and all subgroups:

A. Comparison between the ischemic subgroups as regard LVEF by 2D echo:

We found that 60 patients $(75 \%)$ had reduced LVEF by $2 \mathrm{D}$ echo $(<52 \%$ in males $\&<54 \%$ in females) $\& 20$ patients $(25 \%)$ with preserved LVEF $(\geq 52 \%$ in males $\& \geq 54 \%$ in females) ( $\mathrm{P}$ value 0.001) accordingly we divided the ischemic patients (group I) into 2 subgroups according to their LV EF measured by $2 \mathrm{D}$ echocardiography:

Group A1: Included 60 patients (75\%) (48 males and 12 females) have reduced LVEF by $2 \mathrm{D}$ echo $(<52 \%$ in males \& < $54 \%$ in females) (mean $=46.3 \pm 9.68)$.

All patients with impaired LV EF 2D echo had impaired LV GLS by STE, 46 patients with impaired LV EF 2D echo had impaired function by TDI strain, 54 patients with impaired LV EF 2D echo had impaired average $\mathrm{Sa}$ and only 22 patients with impaired LV EF 2D echo had impaired function by gated SPECT LVEF.

Correlation between 2D LV EF and different parameters:

There was a strongly positive correlation between 2D echo LVEF and STE-GLS at value of $(\mathrm{r}=.747, \quad \mathrm{P}$ value $=0.000), 2 \mathrm{D}$ echo $\mathrm{LVEF}$ and gated SPECT at value of $(\mathrm{r}=.569, \mathrm{P}$ value $=0.000)$ and a moderately positive correlation between 2D LVEF and Avg TDI strain value of $(r=.316, \quad P$ value=0.047), $2 \mathrm{D}$ LVEF and PER value of $(\mathrm{r}=.382$, $\mathrm{P}$ value $=0.015)$, strongly negative correlation between 2D LVEF and GATED SPECT LVESV value of $(r=$ -.568, $\mathrm{P}$ value $=0.000$ ), $2 \mathrm{D}$ LVEF and GATED SPECT LVEDV value of $(r=$ -.546, $\mathrm{P}$ value=0.000), moderately negative correlation between $2 \mathrm{D}$ LVEF and SWMA 2D score value of $(r=-.413, \mathrm{P}$ value $=0.008)$, $2 \mathrm{D}$ LVEF and GATED SPECT SWMA score value of $(r=-.349, \mathrm{P}$ value $=0.027$, (Table 4). 
Table (4): Correlation between 2D echo LV EF and different parameters

\begin{tabular}{|c|c|c|}
\hline 2D LVEF & $\begin{array}{c}\text { Pearson correlation } \\
\text { coefficient }(\boldsymbol{r})\end{array}$ & P value \\
\hline SWMA score & $\mathbf{- 0 . 4 1 3}$ & $\mathbf{0 . 0 0 8}$ \\
\hline Lat Sa & $\mathbf{0 . 4 6 3}$ & $\mathbf{0 . 0 0 3}$ \\
\hline Avg TDI strain & $\mathbf{0 . 3 1 6}$ & $\mathbf{0 . 0 4 7}$ \\
\hline STE GLS & $\mathbf{0 . 7 4 7}$ & $<\mathbf{0 . 0 0 1}$ \\
\hline Avg E & $\mathbf{- 0 . 3 8 6}$ & $\mathbf{0 . 0 4 4}$ \\
\hline Avg A & $\mathbf{- 0 . 3 5 5}$ & $\mathbf{0 . 0 2 5}$ \\
\hline Avg S & $\mathbf{0 . 4 4 4}$ & $\mathbf{0 . 0 0 4}$ \\
\hline Sept Sa & $\mathbf{0 . 3 4 3}$ & $<\mathbf{0 . 0 0 1}$ \\
\hline gated SPECT LVEDV & $\mathbf{- 0 . 5 4 6}$ & $<\mathbf{0 . 0 0 1}$ \\
\hline gated SPECT LVESV & $\mathbf{- 0 . 5 6 8}$ & $<.0001$ \\
\hline gated SPECT LVEF & $\mathbf{0 . 5 6 9}$ & $<.0001$ \\
\hline gated SPECT SWMA score & $\mathbf{- 0 . 3 4 9}$ & $\mathbf{0 . 0 2 7}$ \\
\hline PER & $\mathbf{0 . 3 8 2}$ & $\mathbf{0 . 0 1 5}$ \\
\hline
\end{tabular}

Group A2: Included 20 patients (12 male and 48 female) with preserved LVEF by $2 \mathrm{D}$ echo ( $\geq 52$ in males $\& \geq 54$ in females) (mean $=58.9 \pm 4.68)$.

B. Comparison between the ischemic subgroups as regard STE-GLS:

We divided the ischemic group (group I) as regard STE-GLS into:

Group 1B: Included 74 patients $(92.5 \%)$ (56 males and 18 females) with impaired

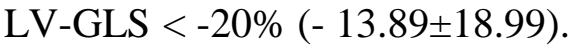

There were 74 patients with impaired STE-GLS had impaired Avg Sa, only 22 patients with impaired STEGLS had impaired gated SPECT LVEF, 60 patients with impaired STE-GLS had impaired LVEF by $2 \mathrm{D}$ echo and 54 patients with impaired STE-GLS had impaired Avg TDI strain.

Correlations between the STE-GLS and different parameters:

There was a strongly positive correlation between the STE-GLS and LVEF by $2 \mathrm{D}$ echo at value of $(\mathrm{r}=.747, \mathrm{P}$ value $=0.000)$, STE-GLS and GATED
SPECTLVEF at value of $(\mathrm{r}=.590, \mathrm{P}$ value=0.000), STE-GLS and Avg $\mathrm{Sa}$ at value of $(\mathrm{r}=.545, \mathrm{P}$ value $=0.000)$, moderately positive correlation between the STE-GLS and FS at value of $(r=.408$, $\mathrm{P}$ value $=0.009)$, STE-GLS and PER at value of $(\mathrm{r}=.387$, $\mathrm{P}$ value $=0.014)$, strongly negative correlation between STE-GLS and GATED SPECTLVESV at value of $(\mathrm{r}=-.575, \mathrm{P}$ value $=0.001)$, STE-GLS and GATED SPECTLVEDV at value of $(\mathrm{r}=$ $.514, \mathrm{P}$ value $=0.001)$, moderately negative correlation between STE-GLS and LVESD at value of $(\mathrm{r}=-.454, \quad \mathrm{P}$ value $=0.003)$, STEGLS and Avg Ea at value of $(r=-.404, P$ value $=0.010)$,STEGLS and SWMA 2D score at value of ( $\mathrm{r}=$ $-.384, \quad \mathrm{P}$ value=0.014), STE-GLS and SWMA GATED SPECT score at value of $(\mathrm{r}=-.367, \mathrm{P}$ value $=0.020)$, STE-GLS and LVEDD at value of $(\mathrm{r}=-.362, \mathrm{P}$ value $=0.022)$, STE-GLS and Avg Aa at value of $(\mathrm{r}=-.364, \mathrm{P}$ value $=0.021)$, (Table 5). 
Table (5): Correlations between the STE-GLS and different parameters

\begin{tabular}{|c|c|c|}
\hline STE GLS & $\begin{array}{c}\text { Pearson correlation } \\
\text { coefficient }(\boldsymbol{r})\end{array}$ & P value \\
\hline SWMA score & $\mathbf{- . 3 8 4 . 0 1 4}$ & $\mathbf{- . 3 8 4 . 0 1 4}$ \\
\hline 2D EF & $\mathbf{0 . 7 4 7}$ & $<\mathbf{0 . 0 0 1}$ \\
\hline FS & $\mathbf{0 . 4 0 8}$ & $\mathbf{0 . 0 0 9}$ \\
\hline Avg E & $\mathbf{- 0 . 4 0 4}$ & $\mathbf{0 . 0 1 0}$ \\
\hline Avg A & $\mathbf{- 0 . 3 6 4}$ & $\mathbf{0 . 0 2 1}$ \\
\hline Avg S & $\mathbf{0 . 5 4 5}$ & $\mathbf{0 . 0 0 1}<$ \\
\hline gated SPECT LVEDV & $\mathbf{- 0 . 5 1 4}$ & $<\mathbf{0 . 0 0 1}$ \\
\hline gated SPECT LVESV & $\mathbf{- 0 . 5 7 5}$ & $\mathbf{0 . 0 0 1}<$ \\
\hline gated SPECT LVEF & $\mathbf{0 . 5 9 0}$ & $<\mathbf{0 . 0 0 1}$ \\
\hline gated SPECT SWMA Score & $\mathbf{- 0 . 3 6 7}$ & $\mathbf{0 . 0 2 0}$ \\
\hline PER & $\mathbf{0 . 3 8 7}$ & $\mathbf{0 . 0 1 4}$ \\
\hline
\end{tabular}

Group 2B: Included 6 patients $(7.5 \%)(4$ males \& 2 female) with preserved LVGLS function $>-20 \%(-21.24 \pm 1.23)$.

C. Comparison between the ischemic subgroups as regard Avg TDI strain:

We divided the ischemic group (group I) as regard Avg TDI strain into:

Group 1C: Included 60 patients (75\%) (44 males and 16 females) with impaired Avg TDI strain <-20\% (- 14.66 \pm 3.3$)$.

Patients with impaired strain by Avg TDI 52 patients with impaired TDI strain had impaired STE-GLS, 20 patients with impaired TDI strain had impaired gated SPECT LVEF, 56 patients with impaired TDI strain had impaired Avg Sa, 46 patients with impaired TDI strain had impaired LVEF by 2D echo.

Correlations between the Avg TDI strain and different parameters:

There was a moderately positive correlation between the Avg TDI strain and gated SPECTLVEF at value of $(\mathrm{r}=.367, \mathrm{P}$ value=0.020), Avg TDI strain and Avg $\mathrm{Sa}$ at value of $(\mathrm{r}=.355, \mathrm{P}$ value $=0.024)$, Avg TDI strain and LVEF BY 2D ECHO at value of $(r=.316, P$ value $=0.047$ ), moderately negative correlation between Avg TDI strain and gated SPECTLVEDV at value of $(\mathrm{r}=$ $.441, \mathrm{P}$ value $=0.004)$, Avg TDI strain and GATED SPECT LVESV at value of $(r=-$ .433, $\mathrm{P}$ value $=0.005)$, Avg TDI strain and Avg Aa at value of $(\mathrm{r}=-.393, \quad \mathrm{P}$ value=0.012), Avg TDI strain and SWMA GATED SPECT score at value of $(r=-$ $.359, \mathrm{P}$ value $=0.023)$, Avg TDI strain and Avg $\mathrm{Ea}$ at value of $(\mathrm{r}=-.325, \quad \mathrm{P}$ value=0.041), Avg TDI strain and LVEDD at value of $(\mathrm{r}=-.347, \quad \mathrm{P}$ value $=0.028)$, Avg TDI strain and LVESD at value of $(r=-.320, P$ value $=0.044)$, (Table 6). 
Table (6): Correlations between the Avg TDI strain and different parameters

\begin{tabular}{|c|c|c|}
\hline Avg TDI strain & $\begin{array}{c}\text { Pearson correlation } \\
\text { coefficient }(\boldsymbol{r})\end{array}$ & P value \\
\hline gated SPECT LVEDV & $\mathbf{- 0 . 4 4 1}$ & $\mathbf{0 . 0 0 4}$ \\
\hline gated SPECT LVESV & $\mathbf{- 0 . 4 3 3}$ & $\mathbf{0 . 0 0 5}$ \\
\hline gated SPECT LVEF & $\mathbf{0 . 3 6 7}$ & $\mathbf{0 . 0 2 0}$ \\
\hline gated SPECT SWMA score & $\mathbf{- 0 . 3 5 9}$ & $\mathbf{0 . 0 2 3}$ \\
\hline Avg Ea & $\mathbf{- 0 . 3 2 5}$ & $\mathbf{0 . 0 4 1}$ \\
\hline Avg Aa & $\mathbf{- 0 . 3 9 3}$ & $\mathbf{0 . 0 1 2}$ \\
\hline Avg Sa & $\mathbf{0 . 3 5 5}$ & $\mathbf{0 . 0 2 4}$ \\
\hline LVEF-2D & $\mathbf{0 . 3 1 6}$ & $\mathbf{0 . 0 4 7}$ \\
\hline LVESD & $\mathbf{- 0 . 3 2 0}$ & $\mathbf{0 . 0 4 4}$ \\
\hline
\end{tabular}

Group 2C: Included 20 patients (25\%) (16 males and 4 female) with preserved function $\geq-20 \%(-22.48 \pm 1.97)$.

D. Comparison between the ischemic subgroups as regard Avg Sa:

We divided the ischemic group (group I) as regard Avg Sa into:

Group 1D: Included 72 patients $(90 \%)$ (52 males and 20 females) with impaired Avg Sa $<7.5 \mathrm{~cm} / \mathrm{s}(5.45 \pm 1.69)$.

Patients with impaired by Avg Sa 64 patients with impaired Avg $\mathrm{Sa}$ had impaired STE-GLS, 20 patients with impaired Avg $\mathrm{Sa}$ had impaired gated (Single Photon Emission Computed Tomography (SPECT) LVEF, 56 patients with impaired Avg Sa had impaired TDI strain, 54 patients with Avg $\mathrm{Sa}$ had impaired LVEF by 2D echo.
Correlations between the Avg Sa and different parameters:

There was a strongly positive correlation between Avg Sa and STE-GLS at value of $(\mathrm{r}=.545, \mathrm{P}$ value $=0.000)$, moderately positive correlation between Avg Sa and LVEF BY 2D ECHO at value of $(r=.444, \mathrm{P}$ value=0.004), Avg $\mathrm{Sa}$ and Avg TDI strain at value of $(\mathrm{r}=.355, \mathrm{P}$ value=0.024), Avg $\mathrm{Sa}$ and gated SPECTLVEF at value of $(\mathrm{r}=.339, \mathrm{P}$ value $=0.033$, moderately negative correlation between Avg Sa and GATED SPECTSWMA score at value of $(r=-.455$, $P$ value $=0.003)$, Avg $S a$ and GATED SPECTLVESV at value of $(\mathrm{r}=-.445, \mathrm{P}$ value=0.004), Avg Sa and SWMA score $2 \mathrm{D}$ at value of $(\mathrm{r}=-.444, \mathrm{P}$ value $=0.012)$, Avg $\mathrm{Sa}$ and GATED SPECTLVEDV at value of $(r=-.443, \mathrm{P}$ value $=0.004)$, (Table 7).

Table (7): Correlations between the Avg Sa and different parameters:

\begin{tabular}{|c|c|c|}
\hline Avg Sa & $\begin{array}{c}\text { Pearson correlation } \\
\text { coefficient }(\boldsymbol{r})\end{array}$ & P value \\
\hline LVEF by 2D echo & $\mathbf{0 . 4 4 4}$ & $\mathbf{0 . 0 0 4}$ \\
\hline SWMA score 2D & $\mathbf{- 0 . 4 4 4}$ & $\mathbf{0 . 0 0 4}$ \\
\hline Avg TDI strain & $\mathbf{0 . 3 5 5}$ & $\mathbf{0 . 0 2 4}$ \\
\hline STE-GLS & $\mathbf{0 . 5 4 5}$ & $<\mathbf{0 . 0 0 1}$ \\
\hline gated SPECT LVEDV & $\mathbf{- 0 . 4 4 3}$ & $\mathbf{0 . 0 0 4}$ \\
\hline gated SPECT LVESV & $\mathbf{- 0 . 4 4 5}$ & $\mathbf{0 . 0 0 4}$ \\
\hline gated SPECTLVEF & $\mathbf{0 . 3 3 9}$ & $\mathbf{0 . 0 3 3}$ \\
\hline gated SPECT SWMA score & $\mathbf{- 0 . 4 5 5}$ & $\mathbf{0 . 0 0 3}$ \\
\hline
\end{tabular}


Group 2D: Included 8 patients (10\%) (8 males) with preserved function $(\geq 7.5$ $\mathrm{cm} / \mathrm{s})(8.7 \pm .73)$.

E. Comparison between the ischemic subgroups as regard gated SPECT LVEF:

We divided the ischemic group (group I) as regard gated SPECT LVEF into:

Group 1E: Included 22 patients $(27.5 \%)$ (20 males and 2 female) with impaired gated SPECT LVEF < 50\% (37.18 \pm 8.86$)$.

Patients with impaired gated SPECT LVEF: 20 patients with impaired gated SPECT LVEF had impaired Avg Sa , 22 patients with impaired gated SPECT LVEF had impaired 2D echo LVEF , 20 patients with impaired gated SPECT LVEF had impaired Avg TDI strain, and all patients with impaired GATED SPECT LVEF had impaired STE-GLS.

Correlations between the gated SPECT LVEF and different parameters:

There was a strongly positive correlation between the gated SPECT LVEF and PER at value of $(r=.814, \mathrm{P}$ value $=0.000$ ), moderately positive correlation between gated SPECT LVEF and Avg $\mathrm{Sa}$ at value of $(\mathrm{r}=.339, \mathrm{P}$ value $=0.033$, strongly negative correlation between gated SPECT LVEF and gated SPECT SWMA score at value of $(\mathrm{r}=-.833, \mathrm{P}$ value $=0.000)$, moderately negative correlation between gated SPECT LVEF and Avg Ea at value of ( $r=$ -.385 , $\mathrm{P}$ value $=0.014)$, (Table 8).

Table (8): Correlations between the gated SPECT LVEF and different parameters:

\begin{tabular}{|c|c|c|}
\hline Gated SPECT LVEF & $\begin{array}{c}\text { Pearson correlation } \\
\text { coefficient }(\boldsymbol{r})\end{array}$ & P value \\
\hline Avg Ea & $\mathbf{- 0 . 3 8 5}$ & $\mathbf{0 . 0 1 4}$ \\
\hline Avg Sa & $\mathbf{0 . 3 3 9}$ & $\mathbf{0 . 0 3 3}$ \\
\hline gated SPECTSWMA score & -0.833 & $<0.001$ \\
\hline PER & $\mathbf{0 . 8 1 4}$ & $<\mathbf{0 . 0 0 1}$ \\
\hline
\end{tabular}

Group 2E: Included 58 patients $(72.5 \%)$ (40 males \& 18 female) with preserved function gated SPECT LVEF > 50\% (63.69 \pm 6.64$)$.

Correlations between the PER and different parameters:

There was a strongly positive correlation between PER and gated SPECT LVEF at value of $(\mathrm{r}=.814, \mathrm{P}$ value $=0.000)$, moderately positive correlation between PER and FS at value of $(\mathrm{r}=.488, \mathrm{P}$ value=0.001), PER and STE-
GLS at value of $(\mathrm{r}=.387, \mathrm{P}$ value $=.014)$, PER and LVEF- 2D at value of $(\mathrm{r}=.382, \mathrm{P}$ value $=.015)$, strongly negative correlation between PER and gated SPECTLVESV at value of $(r=-.736, P$ value $=0.000)$, PER and SWMA 2D score at value of $(r=$ .693, $\mathrm{P}$ value=0.000), PER and gated SPECTLVEDV at value of $(\mathrm{r}=-.635, \mathrm{P}$ value $=0.000$ ), moderately negative correlation between PER and LVESD at value of $(\mathrm{r}=-.478, \mathrm{P}$ value $=0.002)$, (Table 9). 
Table (9): Correlations between the PER and different parameters

\begin{tabular}{|c|c|c|}
\hline PER & $\begin{array}{c}\text { Pearson correlation } \\
\text { coefficient }(\boldsymbol{r})\end{array}$ & P value \\
\hline gated SPECTLVEF & .814 & .000 \\
\hline gated SPECTLVESV & $\mathbf{. 7 3 6}$ & .000 \\
\hline gated SPECTLVEDV & $\mathbf{. 6 3 5}$ & .000 \\
\hline STE-GLS & .387 & .014 \\
\hline SWMA score 2D & $\mathbf{. 6 9 3}$ & .000 \\
\hline FS & .488 & .001 \\
\hline LVEF-2D &. .382 & .015 \\
\hline LVESD & $\mathbf{- 4 7 8}$ & .002 \\
\hline
\end{tabular}

\section{DISCUSSION}

Left ventricular function is one of the most important determinants for prognosis in patients with coronary artery disease (CAD). Patients with impaired LV systolic function represent a high risk group with significantly higher annual mortality than those with preserved LV function, and survival rates decline in proportion to the severity of dysfunction, Ischemia plays a pivotal role in the development and progression of both types of $\mathrm{HF}$ (HFrEF and HFpEF). Patients with coronary artery disease [CAD] are more likely to have HFrEF (rather than HFpEF) as a result of acute ischemic injury causing MI with scar formation. (Islam et al., 2019).

Chronic heart failure due to left ventricular systolic impairment is characterized by a very poor prognosis. A five year mortality of $41.5 \%$ is significantly higher in these patients than in those with preserved systolic function. However, also patients with heart failure and preserved systolic function have $25 \%$ five year mortality due to abnormal autonomic function, Coronary artery disease (CAD) associated with left ventricular systolic dysfunction is a condition related to poor prognosis (Eduardo et al., 2017).

According to ESC guidelines 2019 of chronic coronary syndrome an echocardiographic study will provide important information about cardiac function and anatomy. LV ejection fraction (LVEF) is often normal in patients with CCS (Daly et al., 2013).

A decreased LV function and/or regional wall motion abnormalities may increase the suspicion of ischemic myocardial damage, (Daly et al., 2016) and a pattern of LV dysfunction following the theoretical distribution territory of the coronary arteries is typical in patients who have already had an MI. The detection of regional wall motion abnormalities can challenging by visual assessment, and detection of early systolic lengthening, decreased systolic shortening, or postsystolic shortening by strain imaging techniques might be helpful in patients with apparently normal LV function but with clinical suspicion of CCS (Smedsrud et al., 2012).

STE is a new echocardiographic technique that allows a precise evaluation of myocardial function. This method is accurate, reproducible, and angle independent, and it enables a complete 
assessment of regional and global function in three directions. In contrast, TDI is angle dependent, prone to noises, less accurate, and able to assess limited region of tissue (Mor-Avi et al., 2011).

Strain echocardiography, performed by using the speckle tracking technique, can identify subclinical left ventricular dysfunction before left ventricular ejection fraction declines. Strain echocardiography is a powerful predictor of cardiac mortality and morbidity for numerous cardiac conditions; it provides additional prognostic information over ejection fraction assessment alone (Sushil et al., 2019).

In this study we assessed left ventricular systolic function by different noninvasive imaging modalities in patients with chronic ischemic heart disease. As regard demographic data we found a statistically highly significant differences between group I and group II as regard the following: sex (male), DM, HTN, DLP, smoking, FH. Also, there were statistically significant differences between the two group as regard chest pain, SBP, DBP and ECG abnormality.

These results were concordant to another study for $\mathrm{CV}$ disease risk factors in the Egypt STEP wise survey which was conducted by the Ministry of Health and Population (Epidemiology and Surveillance unit/preventive sector) in collaboration with the WHO office in Egypt in the years 2011-12. It was a representative household survey on persons aged 15-65 years (5080 participants) in 2011- 2012 (Egypt National STEPwise Survey 2011-2012) This survey reported prevalence of hypertension was $38.7 \%$ for men and
$40.8 \%$ for women, prevalence of dyslipidemia was $37.1 \%$ for men and 36.4 $\%$ for women, prevalence of smoking was $46 \%$ for men and $0.4 \%$ for women, and prevalence of DM was $20.7 \%$ for men and $13.3 \%$ for women (Ihab et al., 2014).

The current profile of risk factors is changing expressing a national epidemiologic transition phase towards relative increase in non-communicable diseases which is strongly linked with unhealthy lifestyle (Gersh et al., 2010).

These results were disconcordant to Ehab et al. (2016) who found that there were no statistically significant differences between the control group and ischemic group as regard of age, sex, hypertension, diabetes mellitus, smoking and character of chest pain.

We found statistically significant differences between group I (ischemic patients) and group II (control group) as regard the following echocardiographic parameters LVEDD, LVEF2D echo, SWMA 2D echo score, AO diameter and significant differences between the studied groups as regard: LVESD, LA diameter These results were concordant to Soren et al. (2012) who found that there was a significant difference between the ischemic group and the control group as regard conventional echocardiographic parameters. These results were discordant with the study of Ehab et al. (2016) who found that there was no statistically significant difference between the control group and ischemic group as regard to LVEDD, LVESD, LV EF\% and LVFS\%. Also, we found in our study, that there was a significant difference between group I and group II as regard average Sa. 
Soren et al. (2012) found that there was a difference between the ischemic group and the control group as regard TDI velocity parameters. This result was in agreement with Soren et al. (2010) who found that both global systolic and diastolic performance was reduced, with significantly reduced $\mathrm{Sa}$, significantly increased E/e, and reduced e'. Our results showed that there were significant decrease in STE-GLS in group I compared to group II. This result was concordant to Md Saqif et al. (2018) who found that peak systolic longitudinal strain (both global and segmental) significantly decreased in patients with significant coronary artery disease on coronary angiogram group. Our result was in agreement with Tor Biering et al. (2014) who found that Global longitudinal peak systolic strain was significantly lower in patients with CAD compared with patients without (Soren et al., 2012).

In our study, we found that there was a strong positive correlation between $2 \mathrm{D}$ echo LVEF and STE-GLS. Our results were concordant to Sushil et al. (2019) who found that for patients with impaired LVEF, GLS and LVEF have a linear relationship, with a GLS of $-11 \%$ or $-12 \%$ corresponding to LVEF of $35 \%$, In contrast, GLS and LVEF have a curvilinear relationship in patients with normal LVEF. (Onishi et al., 2015) Therefore, the ability of GLS to detect subclinical myocardial dysfunction is likely greatest for patients with normal LVEF, and the advantage of GLS over LVEF may be its sensitivity to detect early subclinical cardiomyopathy before 2D echo LVEF declines (Sushil et al., 2019).
In our study, we found that there was a strong positive correlation between 2D echo LVEF and gated SPECT. Our results were concordant to Patel et al. (2016) who compared the left ventricular ejection fraction (LVEF), end diastolic (EDV) and end systolic volume (ESV) obtained on 4 h gated rest $201 \mathrm{Tl}$ myocardial perfusion single photon emission computed tomography (SPECT) with those obtained by two-dimensional echocardiography (2$\mathrm{D}$ ECHO) in patients with known or suspected coronary artery disease (CAD). Results suggest that the $4 \mathrm{~h}$ rest gated $201 \mathrm{Tl}$ study gives a reliable value for the LVEF compared to 2-D ECHO and can be used in routine clinical practice (Patel et al., 2016).

Also, our results were in agreement with Walid et al. (2010) who found that quantitative gated SPECT, using Tl-201 tracer has a good correlation with echocardiography for the measurements of absolute LV volumes and LVEF (Walid et al., 2010).

Our results were in agreement with Nadish et al. (2016) who found that correlation between gated SPECT and echocardiography with respect to EDV, ESV and LVEF ranged from good to excellent whether we used the post stressor a separate rest acquisition, The same result also was considered by Mohamed et al. (2012) who found that the TDI examination the $S$ velocity was a good simple method for determining LV systolic function as $\mathrm{EF}$ and had significant correlation with biplane EF. In our study we found that there was significant inverse correlation between 2D LVEF and SWMA 2D. This result was concordant to Ismail et al. (2015) who found statistically 
significant inverse correlation between WMSI and EF.

In our study, we found that there was a moderate negative correlation between STE-GLS and SWMA gated SPECT score. This result was reported by Seyhan et al. (2014) who found that there was a negative moderate significant correlation between global $\mathrm{S}$ and SR in EC and LVWMSI in GSPECT. In our study, we found that there was a strongly positive correlation between STE-GLS and Avg Sa.

This result was to Modesto et al. (2016) who found that in patients with cardiomyopathy and myocardial infarction, strain and SR obtained using DSI and 2D STE were strongly correlated. Also, we found that 11 patients were impaired LVEF by gated SPECT while 30 patients were impaired LVEF by 2D echocardiography, this finding explained by in patients with small LV cavities, the LV ESV may be underestimated. This occurs because there is blurring of the LV cavity border due to the relative poor resolution of the gamma camera compared with LV wall thickness, and because of an increase in myocardial count density with contraction, LVEF will be overestimated because of underestimation of the ESV. LVEF is often overestimated in women because women tend to have smaller LV cavities.

These results were concordant to Walid et al. (2010) who found that slightly lower values for LVEF calculated by gated SPECT than by 2D echocardiography. In our study we found that $92.5 \%$ of patients were impaired left ventricular systolic function assessed by speckle tracking echocardiography. This indicates high sensitivity of STE in assessment of LV systolic function. The myocardial fibers most susceptible to ischemia are the longitudinally orientated fibers which are located subendocardially. Measurements of longitudinal motion and deformation are therefore the most sensitive markers of CAD (Soren et al., 2012).

In our study, we found that $90 \%$ of patients were impaired left ventricular systolic function assessed by TDI velocity. TDI velocities can improve the diagnostics of CAD in patients suspected of stable angina pectoris. However, local myocardial velocities obtained by TDI have the disadvantage of being influenced by heart movement and tethering to adjacent segments, which makes

2DSE more suitable for diagnosing impaired segmental longitudinal mechanics caused by CAD (Urheim et al., 2010).

\section{CONCLUSION}

All methods for assessment of left ventricular systolic function are comparable to each other. STE is considered the most sensitive method in assessment of subclinical LV systolic dysfunction and is considered superior to other methods. TDI velocity is highly sensitive methods for assessment of LV systolic function but, it have the disadvantage of being influenced by heart movement and tethering to adjacent segments.

\section{Recommendation}

2D STE should be considered as a routine investigation in the assessment of patients with chronic ischemic heart disease for the early detection of 
asymptomatic LV dysfunction and to guide the best way for management of these group of patients to avoid further progression and deterioration of $\mathrm{LV}$ systolic function.

\section{REFERENCES}

1. Becker D and Merkely B. (2012): Acute coronary syndrome - Orv Hetil., 153(51):2012-15.

2. Daly CA, De Stavola B and Sendon JL. (2016): Euro Heart Survey Investigators. Predicting prognosis instable angina- results from the Euro heart survey of stable angina: prospective observational study. BMJ, 332:262-267.

3. Daly C, Norrie J and Murdoch DL. (2013): TIBET (Total Ischaemic Burden European Trial) study group. The value of routine noninvasive tests to predict clinical outcome instable angina. Eur Heart J, 24:532-540.

4. Eduardo G, Gomes L, Lima P, Felipe P, Jaime L and Filho A. (2017): Ischemic left ventricle systolic dysfunction: An evidencebased approach in diagnostic tools and therapeutics Rev Assoc Med Bras., 63(9):793800.

5. Ehab E, Ibraheem $\mathbf{Y}$ and Moustafa $\mathbf{E}$. (2016): Role of 2-dimensional speckle tracking echocardiography in diagnosis of coronary artery stenosis instable angina pectoris patients Research Article. Interv. Cardiol., 8(6); 731-738.

6. Gersh B.J., Sliwa K. and Mayosi B.M. (2010): The epidemic of cardiovascular disease in the developing world: global implications. Eu H J, 31: 642-8.

7. Islam Y, Elgendy A, Dhruv H, Mahtta N and Carl J. (2019): Medical Therapy for Heart Failure Caused by Ischemic Heart Disease. Circ Res ;124:1520-1535.

8. Ismail A, Samy W, Aly A and Atia A. (2015): Longitudinal strain in patients with STEMI using speckle tracking echocardiography. Correlation with peak infarction mass and ejection fraction. The
Egyptian Journal of Critical Care Medicine, 3:45-53.

9. Migrino R, Zhu $X$ and Pajewski N. (2015): Assessment of segmental myocardial viability using regional 2- dimensional strain echocardiography. J Am Soc Echocardiogr., 20:342-51.

10. Modesto KM, Cauduro $S$ and Dispenzieri A. (2016): Two dimensional acoustic pattern derived strain parameters closely correlate with one-dimensional tissue Doppler derived strain measurements, Eur J Echocardiogr., $7: 315-321$.

11. Mor-Avi V, Lang R and Badano L. (2011): Current and evolving echocardiographic techniques for the quantitative evaluation of cardiac mechanics: ASE/EAE consensus statement on methodology and indica- tions endorsed by the Japanese Society of Echocardiography. Eur J Echocardiogr., 12:167-205.

12. Mujtaba S and Peña JM. (2013): Publication trends in noninvasive cardiovascular imaging: 1991-2011: A retrospective observational study. Am J Cardiovasc Dis., 3:247-54.

13. Patel C, Nadig $M$ and Kurien S. (2016): Left ventricular ejection fraction and volumes on rest gated 201Tl perfusion SPECT: comparison with two dimensional echocardiography. Nucl Med Commun., 27(5):425-9.

14. Seyhan A, Karacavus M, Ahmet E, Celik R and Tutus N. (2014): A Comparison between Quantitative Gated Myocardial Perfusion Scintigraphy and Strain Echocardiography as Indicators of Ventricular Functions in Patients with Anterior Myocardial Infarction. World J Nucl Med., 13(3): 184-189.

15. Smedsrud M, Sarvari $S$ and Haugaa $K$. (2012): Duration of myocardial early systolic lengthening predicts the presence of significant coronary artery disease. J Am Coll Cardiol., 60:1086-1093.

16. Soren H, Hoffmann N, Jan S, Jensen Y, Allan A, Zeeberg $H$ and Iversen M. (2012): Tissue Doppler echocardiography improves the diagnosis of coronary artery stenosis 
instable angina pectoris. European Heart Journal Cardiovascular Imaging, 13: 724-729.

17. Stanton T, Leano $R$ and Marwick TH. (2019): Prediction of all, cause mortality from global longitudinal speckle strain: comparison with ejection fraction and wall motion scoring. Circ Cardiovasc Imaging, 2:356-364.

18. Sushil A. Luis M, Jonathan G, Chan R, Patricia A and Pellikka N. (2019): Echocardiographic Assessment of Left Ventricular Systolic Function: An Overview of Contemporary Techniques, Including
Speckle- Tracking Echocardiography Mayo Clin Proc., 94(1):125-138

19. Urheim $S$, Edvardsen $T$ and Torp $H$. (2010): Myocardial strain by Doppler echocardiography. Validation of a new method to quantify regional myocardial function. Circulation, 102, 1158-1164.

20. Walid O, Mohamrd A and Reda A. (2010): Comparison between Gated SPECT and Echocardiography in Evaluation of Left Ventricular Ejection Fraction. Journal of the Egyptian Nat. Cancer Inst., 12 (4) 301- 306. 


\section{تقييم الوظيفة الإنقباضية للبطين الأيسر بو اسطة الوسائل الغير

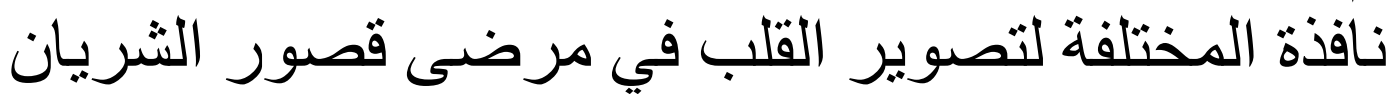

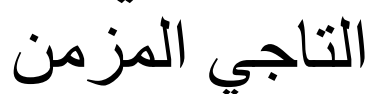

\section{تغزيد أحمد عبد الرحمن، منى حسن نعيم}

قسم القلب والأوعية الاموية، كلية الطب (بنات)، جامعة الأزهر

E-mail: tahersalman2017@gmail.com

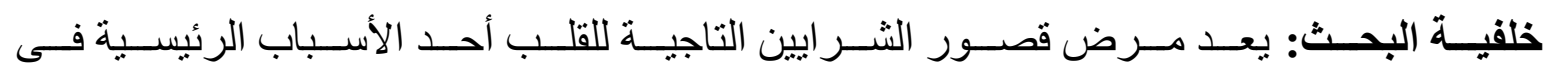

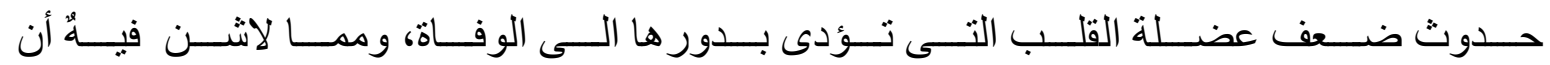

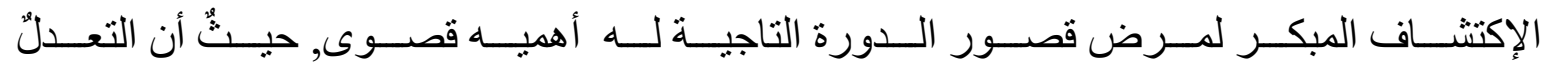

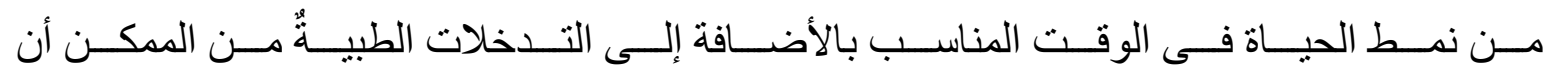

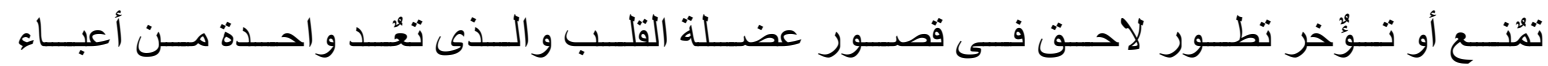

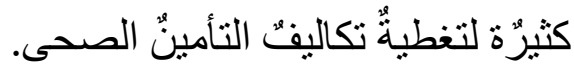

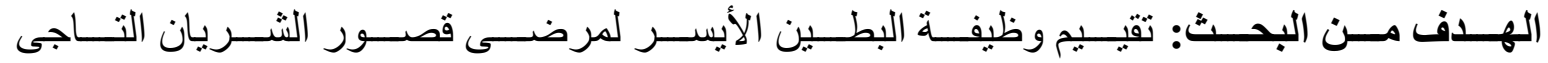

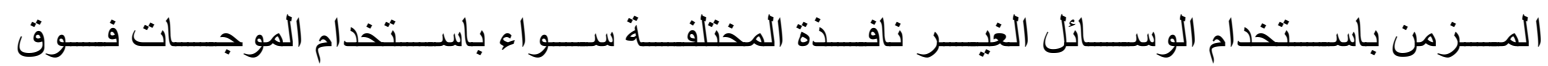
الصوتية أو عن طريق المسح الذرى لعضلة القلب.

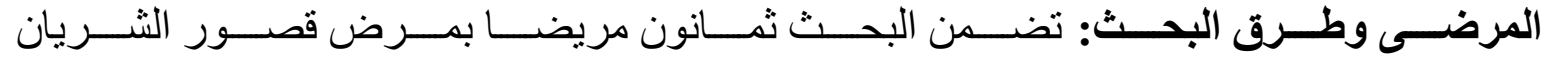

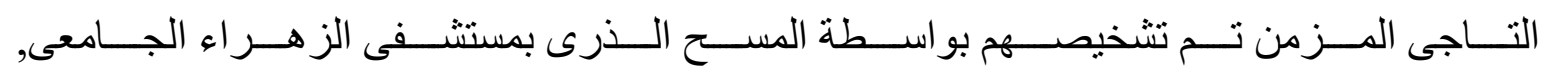

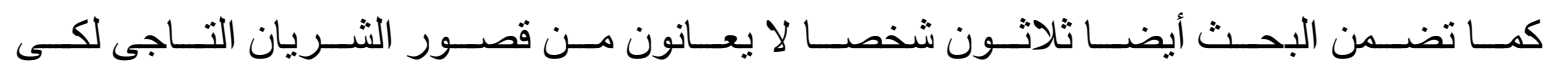
ينم مقارنة تقييم وظيفة البطين الأيسر للمرضى بمثيلتها للأصحاء. تم تقيم وظيفة البطين الأيسر للمرضى والأصحاء بالطرق التالية:

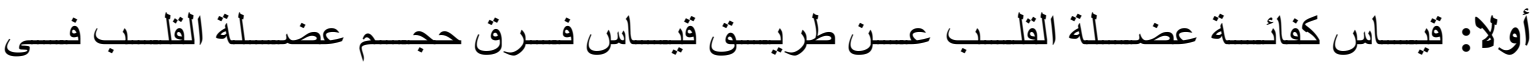
الإنقباض و الإنبساط.

ثانيا: قياس كفائة عضلة القلب باستخدام تقنية الدوبلر النسيجى لعضلة القلب. ثالثا: قياس كفائة عضلة القلب باستخدام التتبع النقطى ثنائى الأبعاد لعضلة القلب. 
رابعا: قياس كفائة عضلة القلب باستخدام المسح الذرى لعضلة القلب.

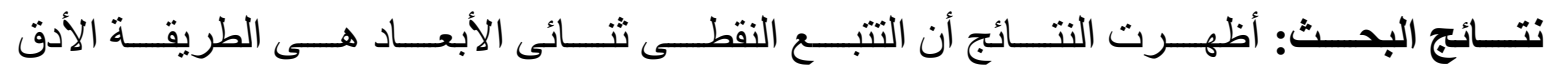

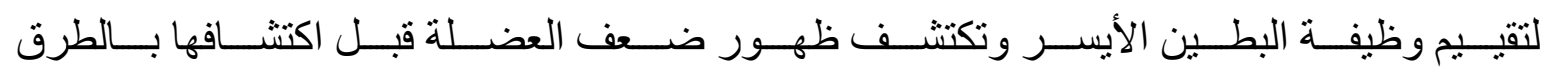
الأخرى.

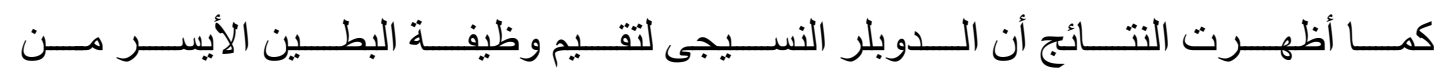
الطرق المتميزة أيضا فى تقييم وظيفة عضلة القلب.

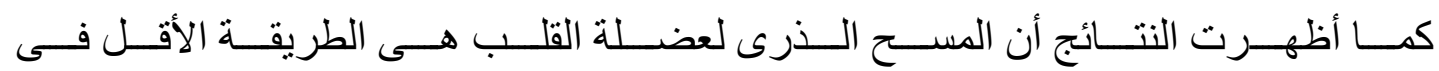

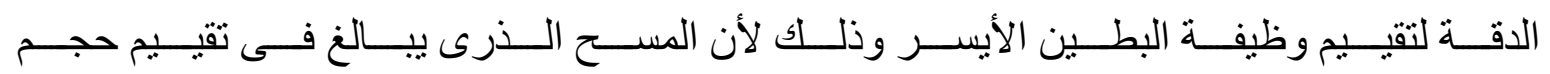

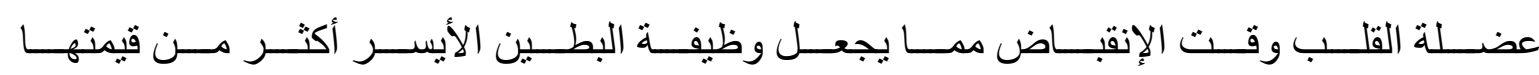
الفعلية.

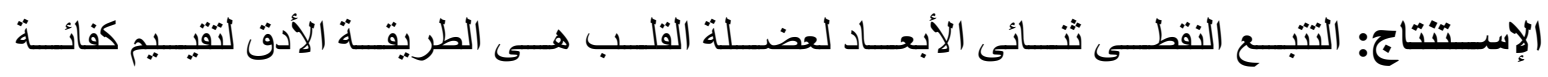
البطين الايسر.

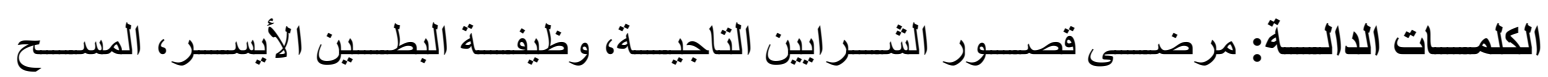
الذرى لعضلة القلب، التتبع النقطى ثنائى الابعاد. 